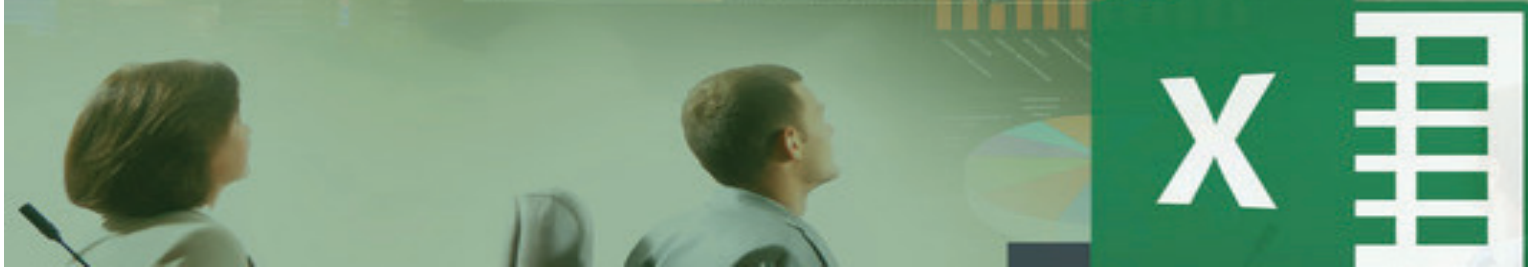

\title{
Desarrollo de un modelo predictivo en Excel para el proceso de inyección de agua utilizando el método de Dake-Welge para un reservorio homogéneo de estrato simple
}

\author{
Development of a predictive model in excel for the water injection process using \\ the method of Dake-Welge for a single layer homogenous reservoir.
}
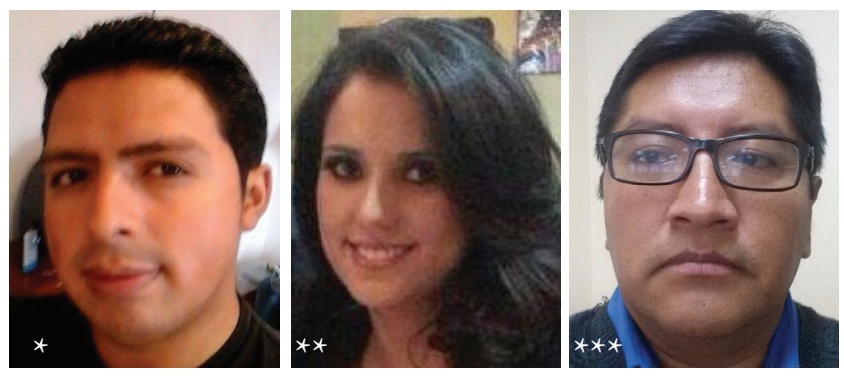

*Joe Navarrete
**Pamela Montalvo
Ingenieros en Petróleos (UCE)
***José Cóndor
Tutor de trabajo de titulación
jacondor@uce.edu.ec

Resumen

El modelo predictivo utiliza el método Dake-Welge para un reservorio homogéneo de estrato simple considerando flujo segregado y flujo difuso. El módulo forma parte de un proyecto para construir un software dedicado al análisis de tecnologías de recuperación secundaria de petróleos. El desarrollo del modelo se realizó en tres etapas: la primera, consistió en la revisión bibliográfica en el tema de inyección de agua; en la segunda, se creó un libro Excel con los fundamentos teóricos, y en la etapa final, se realizó una comparación de resultados generados en Excel con el software WaterDrive. Los resultados obtenidos en el flujo tipo segregado empleando el modelo predictivo fueron similares a los generados en el software WaterDrive. Los resultados obtenidos en el flujo tipo difuso tuvieron un error máximo del 1,2 por ciento para el tiempo de ruptura. Estos resultados llevan a concluir que el modelo predictivo es robusto y estaría listo para probarse en un proyecto piloto. La decisión de desarrollar el modelo usando Excel, permite que pueda ser escrita empleando cualquier lenguaje de programación. Los resultados positivos obtenidos con esta metodología dan confianza en que se puede replicar software comerciales desarrolladas en universidades ecuatorianas.

Palabras clave: recuperación secundaria de petróleo; Inyección de agua; método Dake-Welge; flujo segregado; flujo difuso; excel

\section{Abstract}

This predictive model uses the Dake-Welge method for a homogeneous reservoir of a single layer taking into account the segregated flow and the diffusive flow patterns. This module is part of a project to build an analysis software for technologies of secondary oil recovery. The development of the model was done in three stages: The first s tage consisted in I iterature r eview mainly of specialized b ooks of $w$ aterflooding. The second stage comprised the creation of an Excel workbook with the theoretical fundamentals. Finally, during the third stage there was a validation of the methodology by comparing the results of the Excel workbook with the commercial software WaterDrive owned. The results obtained in the segregated flow pattern of the model matched the outcomes of the software WaterDrive. The results obtained in the diffuse flow pattern had a maximum error of 1.28 per cent in the breakthrough time. These results probe that the model is robust and it would be ready to test in a pilot project.The decision of developing a methodology using Excel allows the writing of it using any programming language. The positive records obtained from this model give confidence that Ecuadorian universities can replicate commercial software at very low costs. 


\section{Introducción}

La técnica más común para mantener la presión de reservorio es inyectar agua. Se estima que los factores de recobro mediante recuperación secundaria están en el rango del 15 al 25 por ciento (Zitha, Felder, Zornes, Brown, \& Mohanty, 2011).

Los campos petroleros en Ecuador han sido explotados mediante métodos primarios, incluyendo levantamiento artificial. Existen $\mathrm{m}$ uy pocos proyectos $\mathrm{d} e$ inyección $\mathrm{d} \mathrm{e}$ agua en el país y como consecuencia no se ha desarrollado tecnologías en el sector público incluida la academia (Condor, Pinto, Achig, \& Romo, 2015).

En contraste, la tecnología de recuperación secundaria mediante inyección de agua ha tenido enormes progresos, especialmente en Norteamérica y Europa donde se han generado importantes publicaciones científicas. Parte de estas publicaciones han descrito automatización de procesos en forma de softwares. Un ejemplo es el software WaterDrive desarrollado por Petroleum Solutions y que está disponible comercialmente (Petroleum Solutions, 2016). El objetivo de la metodología presentada es replicar las características del software WaterDrive.

\section{Material y métodos}

Las etapas seguidas para el desarrollo de la metodología se ilustran en la tabla 1 (Montalvo \& Navarrete, 2016).

Tabla 1

\begin{tabular}{|c|c|c|}
\hline & & Productos \\
\hline 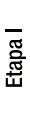 & $\begin{array}{l}\text { Revisión } \\
\text { Bibliográfica }\end{array}$ & $\begin{array}{l}\text { - Propiedades petrofisicas y de } \\
\text { fluidos } \\
\text { - Conceptos fundamentales de } \\
\text { inyección de agua }\end{array}$ \\
\hline 总 & $\begin{array}{l}\text { Construcción de } \\
\text { libro Excel }\end{array}$ & $\begin{aligned} & \text { Flujo fraccional } \\
& \text { - Avance frontal } \\
& \text { - Flujo difuso y flujo segregado } \\
& \text { Método Dake - Welge }\end{aligned}$ \\
\hline 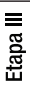 & $\begin{array}{l}\text { Validación de la } \\
\text { metodología }\end{array}$ & $\begin{array}{l}\text { - Comparación de resultados con } \\
\text { software WaterDrive } \\
\text { - Porcentaje de diferencia }\end{array}$ \\
\hline
\end{tabular}

Etapas en el desarrollo de la metodología del modelo predictivo

La principal fuente bibliográfica para la metodología se basó en el libro "The Practice of Reservoir Engineering" de Laurie P. Dake (Dake, 2001). Este libro describe los conceptos clásicos de inyección de agua tales como el flujo fraccional y la ecuación de avance frontal. Este mismo libro es la base del software WaterDrive de Petroleum Solutions.

El libro Excel realiza cálculos usando las fórmulas descritas y las funciones de los diagramas de flujo descritas.
Los tipos de cálculo que la metodología realiza son para flujo difuso y flujo segregado. El flujo difuso considera que las fuerzas viscosas o dinámicas son más dominantes que las fuerzas gravitacionales de modo que las variaciones verticales en la saturación pueden ser ignoradas. Las características típicas de este tipo de condición de flujo son baja permeabilidad vertical combinada con un alto gradiente de presión horizontal. El flujo segregado es el inverso al flujo difuso y se presenta con más frecuencia en reservorios. Las características típicas de este tipo de flujo son alta permeabilidad vertical combinado con un bajo gradiente de presión horizontal (Dake, 1998).

Luego de desarrollar el libro Excel se procedió a la validación de la metodología mediante la comparación de resultados con los obtenidos usando el software WaterDrive. Los resultados obtenidos en el flujo difuso presentaron un error máximo del 1,28 por ciento con el tiempo de ruptura. Los resultados fueron idénticos para el flujo segregado.

\section{Resultados}

\section{Datos de ingreso y resultados esperados}

Los datos de ingreso necesarios para la metodología están resumidos en la Tabla 2.

Tabla 2

Datos de ingreso

\begin{tabular}{|c|c|c|}
\hline Parámetro & Símbolo & Unidades \\
\hline Densidad del petróleo & $\rho_{o}$ & $\mathrm{lb} / \mathrm{ft}^{3}$ \\
\hline Densidad del agua & $\rho_{w}$ & $\mathrm{lb} / \mathrm{ft}^{3}$ \\
\hline Viscosidad del petróleo & $\mu_{o}$ & $\mathrm{cP}$ \\
\hline Viscosidad del agua & $\mu_{w}$ & $\mathrm{cP}$ \\
\hline Factor volumétrico inicial del petróleo & $\beta_{o i}$ & rb/stb \\
\hline Factor volumétrico del petróleo & $\beta_{o}$ & rb/stb \\
\hline Factor volumétrico del agua & $\beta_{w}$ & rb/stb \\
\hline $\begin{array}{l}\text { Permeabilidad relativa del petróleo al } \\
\text { final de la inyección }\end{array}$ & $k_{r o}^{*}$ & fracción \\
\hline $\begin{array}{l}\text { Permeabilidad relativa del agua al final } \\
\text { de la inyección }\end{array}$ & $k_{r w}^{*}$ & fracción \\
\hline Saturación de agua connata & $S_{w c}$ & fracción \\
\hline Saturación de petróleo residual & $S_{\text {or }}$ & fracción \\
\hline Porosidad & $\varnothing$ & fracción \\
\hline Permeabilidad & $k$ & $\mathrm{mD}$ \\
\hline Espesor del reservorio & $e$ & $\mathrm{ft}$ \\
\hline Longitud del reservorio & $L$ & $\mathrm{ft}$ \\
\hline Ancho del reservorio & $A$ & $\mathrm{ft}$ \\
\hline Inclinación del reservorio & $\theta$ & grados \\
\hline Caudal de inyección & $q_{i}$ & stb/d \\
\hline Incremento de saturación de agua & $\Delta S_{w c}$ & fracción \\
\hline $\begin{array}{llll}\text { Incremento del agua acumulada } \\
\text { inyectada }\end{array}$ & $\Delta W_{i d}$ & fracción \\
\hline Exponente de Corey para petróleo & $N_{o}$ & adimensional \\
\hline Exponente de Corey para agua & $N_{w}$ & adimensional \\
\hline Fecha inicio de inyección & & días \\
\hline Fecha final de inyección & & días \\
\hline
\end{tabular}




\section{Ecuaciones usadas}

En la metodología se emplearon las siguientes ecuaci ones:

Petróleo original en sitio (00IP)

$$
O O I P=\frac{A \cdot T \cdot 615 \beta \_o i}{5,615 C)}
$$

Donde: $A$ = ancho del reservorio ( $\mathrm{ft}$ )

$\mathrm{L}=$ longitud del reservorio ( $\mathrm{ft}$ )

$\mathrm{e}=$ espesor del reservorio (ft)

$\varnothing=$ porosidad (fracción)

S_wc = saturación de agua connata (fracción)

$\beta \_$_oi = factor volumétrico del petróleo (rb/stb)

- Relación de movilidad final (M)

$$
M=\frac{\frac{k_{r w}}{\mu_{w}}}{\frac{k_{r o}}{\mu_{o}}}
$$

Donde: $\mathrm{k} \_r w=$ permeabilidad relativa del agua

$\mu \_w=$ viscosidad del agua (CP)

k_ro $=$ permeabilidad relativa del petróleo

$\mu \_o=$ viscosidad del petróleo (cP)

- Relación de movilidad en el frente de choque (M_s )

$$
M_{s}=\frac{\frac{k_{r o} \cdot S_{w f}}{\mu_{o}}+\frac{k_{r o} \cdot S_{w f}}{\mu_{w}}}{\frac{k_{r o}}{\mu_{o}}}
$$

Donde:

S_wf = saturación de agua en el frente de choque

- Exponente Corey del petróleo (N_o )

Donde: S_w = saturación de agua

S_or = saturación de petróleo residual

S_wc = saturación agua connata

- Exponente Corey del agua (N_w )

$$
N_{W}=\frac{\log \left(\frac{S_{w^{-}} S_{w c}}{1-S_{w c^{-}} S_{o r}}\right)}{\log \left(k_{r w}\right)}
$$

\section{- Permeabilidad relativa del agua (k_rw )}

Donde: $\mathrm{k} \_r \mathrm{w}^{\wedge}{ }^{\star}=$ Permeabilidad relativa del agua al final de la inyección

$$
k_{r w}=k_{r w}^{*}\left(\frac{S_{w}-S_{w c}}{1-S_{w c}-S_{o r}}\right)
$$

\section{- Permeabilidad relativa del petróleo (k_ro )}

$$
k_{r o}=k_{r o}^{*}\left(\frac{1-S_{w}-S_{o r}}{1-S_{w c}-S_{o r}}\right)^{N_{o}}
$$

Donde: N_o = Exponente de Corey para petróleo

k_ro $\mathrm{o}^{\wedge *}=$ Permeabilidad relativa del petróleo al final de la inyección

$$
f_{w}=\frac{1}{1+\frac{\mu_{w}}{\mu_{o}} \frac{k_{r o}}{k_{r w}}}
$$

- Variación de la saturación ᄀᄀde agua en reservorio ( $\left.\Delta S \_w e\right)$

$$
\Delta S_{w e}=S_{w e_{i+1}}-S_{w e_{i}}
$$

Donde: $S_{w e_{i+1}}=$ Saturación de agua en tiempo i+1

$$
S_{w e_{i}}=\text { Saturación inicial de agua ( } \mathrm{t}=0 \text { ) }
$$

- Variación de flujo fraccional en reservorio ( $\Delta \mathrm{f} \_$we $)$

$$
\Delta f_{w e}=f_{w e_{i+1}}-f_{w e_{i}}
$$

Donde: $f_{w e_{i+1}}=$ flujo fraccional en tiempo i+1

$$
f_{w e_{i}}=\text { flujo fraccional inicial }(t=0)
$$

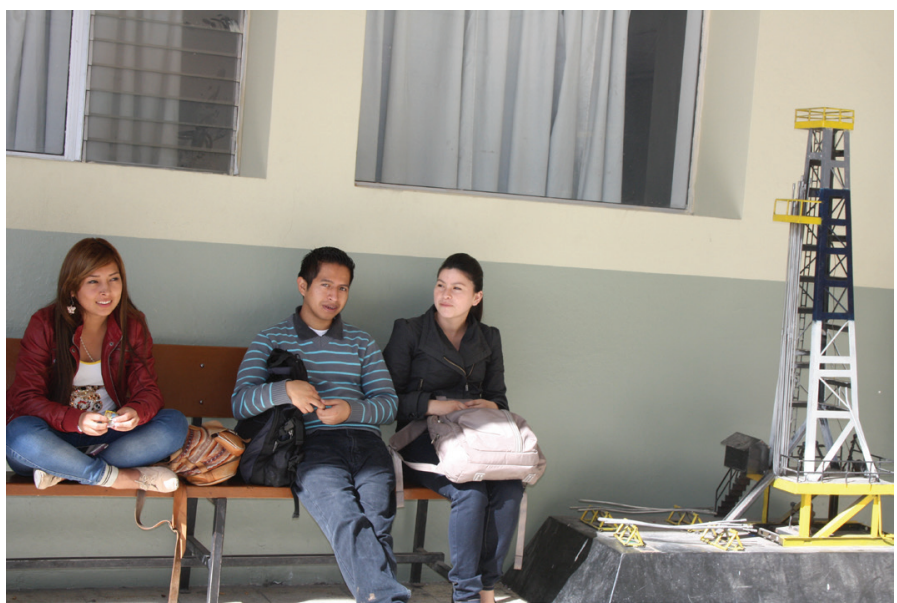


- Variación de flujo fraccional en reservorio $\left(\Delta \boldsymbol{f}_{w e}\right)$

$\Delta f_{w e}=f_{w e_{i+1}}-f_{w e_{i}}$

Donde: $f_{w e_{i+1}}=$ flujo fraccional en tiempo i+1

$f_{w e_{i}}=$ flujo fraccional inicial $(\mathrm{t}=0)$

- Agua inyectada acumulada adimensional $\left(w_{i d}\right)$

$w_{i d}=\frac{1}{\frac{\Delta f_{w e}}{\Delta S_{w e}}}$

- Saturación de agua promedio en reservorio $\left(\overline{S_{w e}}\right)$

$\overline{S_{w e}}=\frac{S_{w e_{i+1}}-S_{w e_{i}}}{2}$

- Flujo fraccional promedio en reservorio $\left(\overline{f_{w e}}\right)$

$\overline{f_{w e}}=\frac{f_{w e_{i+1}}-f_{w e_{i}}}{2}$

- Recuperación de petróleo acumulado adimensional $\left(N_{p d}\right)$

$N_{p d}=\left(\overline{S_{w e}}-S_{w c}\right)+w_{i d} \cdot\left(1-\overline{f_{w e}}\right)$

- Recuperación de petróleo acumulado adimensional $(t)$

$t=\frac{w_{i d} \cdot A \cdot e \cdot L \cdot \emptyset}{5,615 \cdot q_{i}}$

- Tasa de inyección ( $q$ )

$q=t \cdot q_{i}$

- Flujo fraccional en superficie $\left(f_{w s}\right)$

$f_{w s}=\frac{1}{1+\frac{\beta_{W}}{\beta_{O}}\left(\frac{1}{f_{w e}}-1\right)}$

- Caudal de petróleo $\left(q_{o}\right)$

$q_{o}=\frac{q_{w i}}{\beta_{O}+\left(\frac{\beta_{w} \cdot f_{w s}}{1-f_{w s}}\right)}$

Donde: $q_{w i}=$ caudal inyección de agua

- Factor de recuperación (FR)

$F R=\frac{N_{p d}}{O O I P}$
- Caudal de agua $\left(\boldsymbol{q}_{w} \boldsymbol{\beta}_{w}\right)$

$q_{w} \beta_{w}=q_{w i}-q_{o} \beta_{o}$

- Relación agua-petróleo (WOR)

$W O R=\frac{q_{w}}{q_{o}}$

- Corte de agua (BSW)

$B S W=\frac{q_{w}}{q_{o}+q_{w}}$

- Agua inyectada acumulada $\left(W_{i d n}\right)$

$W_{i d n}=\frac{1}{M}+\Delta W_{i d}$

- Transformación (MOV)

$M O V=P V\left(1-S_{o r}-S_{w c}\right)$

- Tiempo de ruptura $\left(t_{b t}\right)$

$t_{b t}=\frac{w_{i d} \cdot A \cdot e \cdot L \cdot \emptyset}{5,615 \cdot q_{i}}$

- Recuperación de petróleo después del tiempo de ruptura $\left(N_{p d}\right)$

$N_{p d}=\left(S_{w e}-S_{w c}\right)+W_{i d} \cdot\left(1-f_{w e}\right)$

- Tasa crítica para by-passing $\left(\boldsymbol{q}_{c}\right)$

$q_{c}=\frac{4.9 \times 10^{-4} \cdot k \cdot k_{r w}^{*} \cdot A \cdot \Delta \gamma \cdot \sin \theta}{\mu_{w}(M-1)}$

- Recuperación de petróleo acumulado

$$
\begin{gathered}
N_{p d}=\left(\frac{1}{M-1}\right) 2 W_{i d} M\left(1-\frac{G}{M-1}\right)\left(1-\frac{W_{i d} G}{M-1}\right. \\
\left.-W_{i d}\right)\left(1-\frac{M+1}{M-1}\right)(G-1)
\end{gathered}
$$

- Recuperación de petróleo

$N_{p d(b t)}=\left(\frac{1}{M-G}\right)$

- Recuperación de petróleo

$W_{i d(\max )}=\left(\frac{M}{G+1}\right)$

- Recuperación de petróleo segregado MOV

$N_{p d i}=\frac{1}{M-1}\left(2 \sqrt{W_{i d i}}-W_{i d i}-1\right)$ 


\section{Diagramas de flujo}

Los siguientes ilustran las relaciones entre las fórmulas y los procesos de la metodología.

\section{a) Diagrama general}

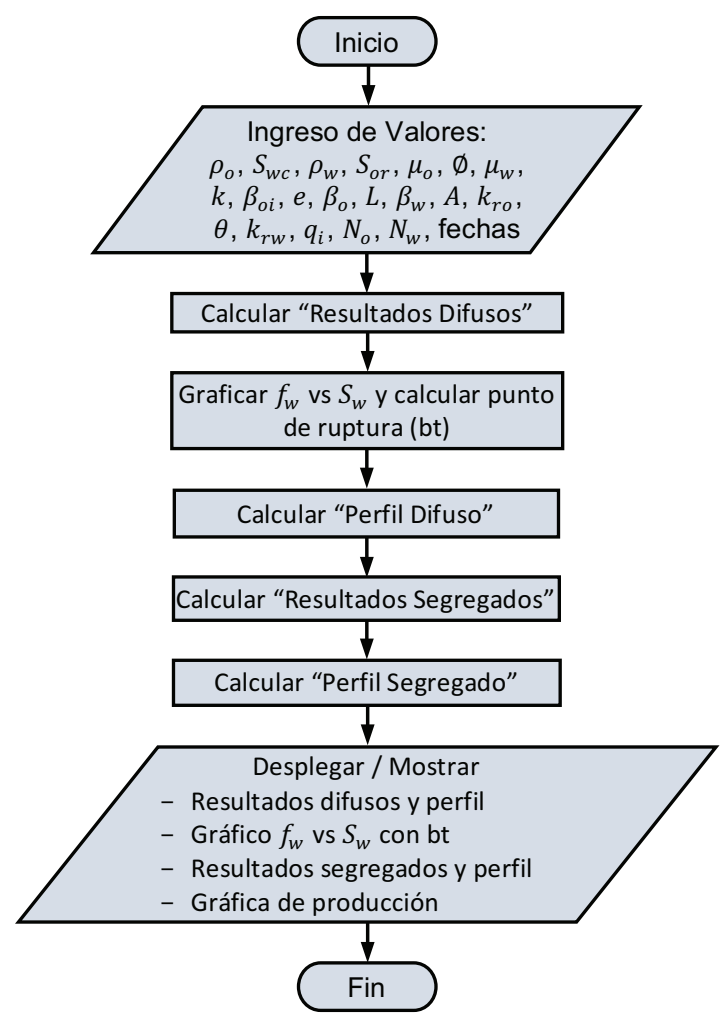

\section{b) Flujo difuso}

\section{- Saturación de agua $\left(S_{w e}\right)$}

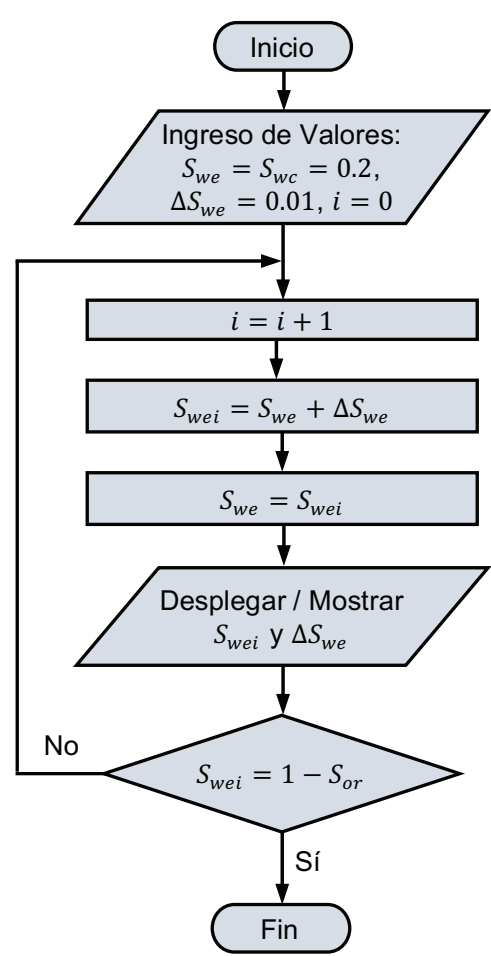

- Permeabilidades relativas $\left(\boldsymbol{k}_{r o}, \boldsymbol{k}_{r w}\right)$

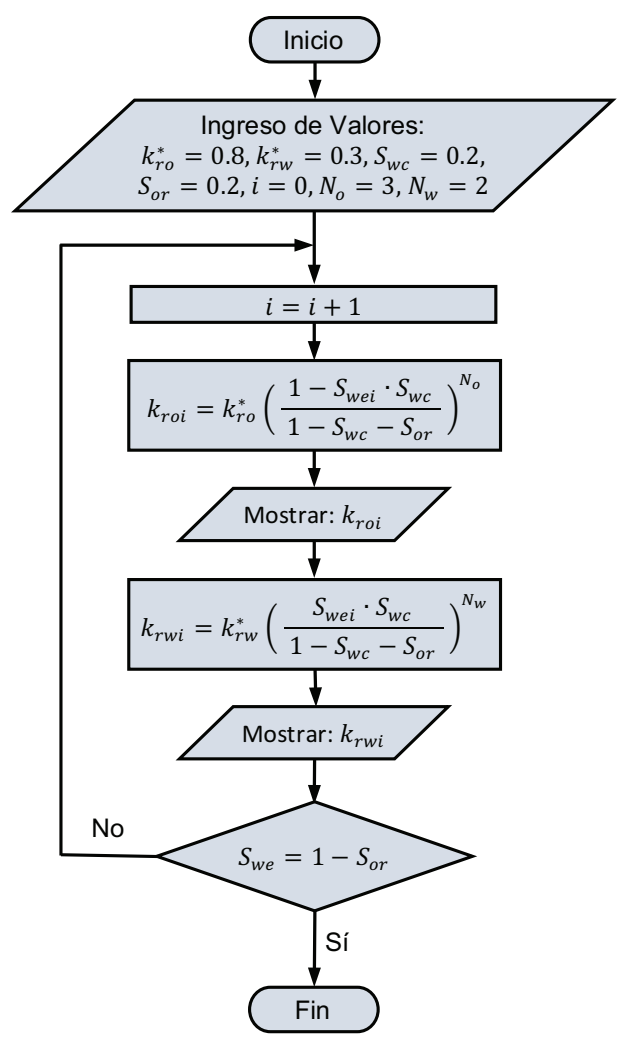

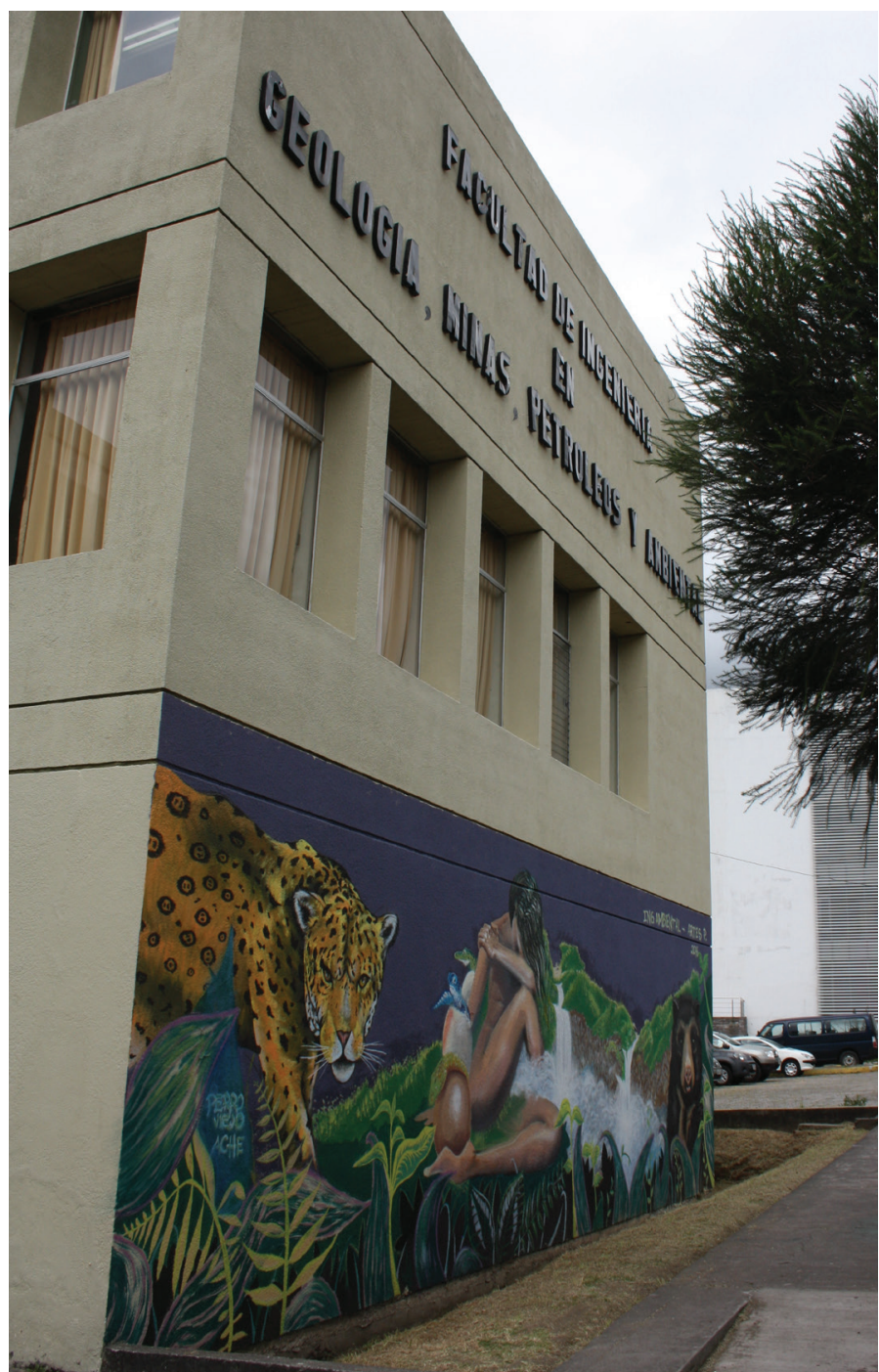


- Permeabilidades relativas $\left(\boldsymbol{k}_{r o}, \boldsymbol{k}_{r w}\right)$

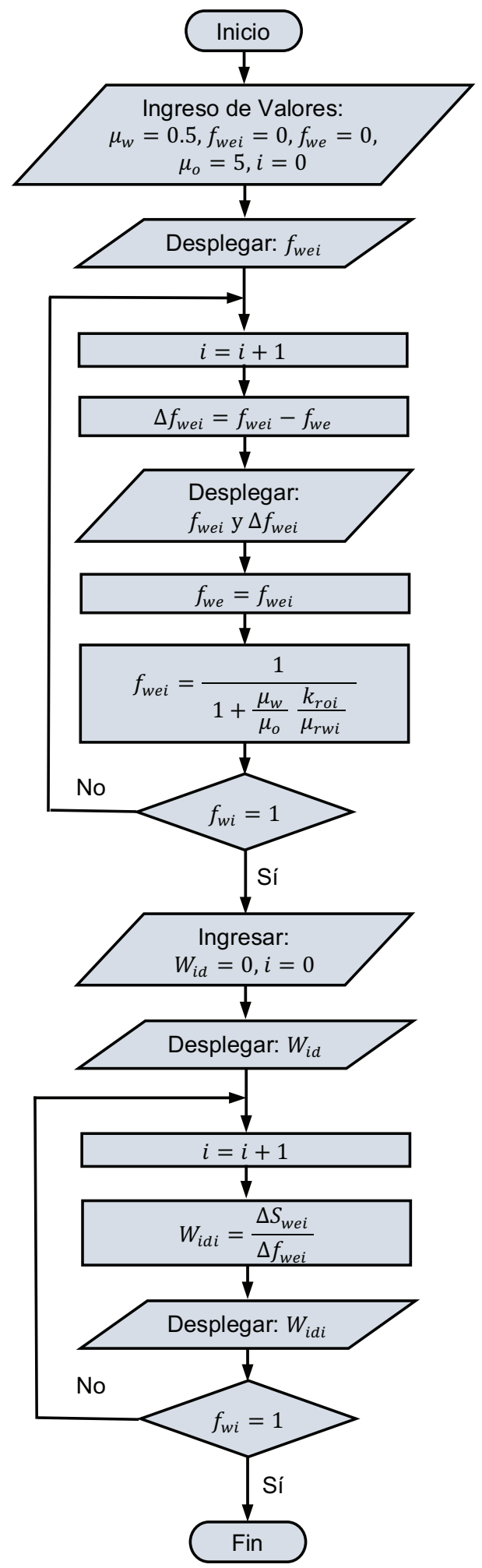

- Saturación y flujo fraccional promedio $\left(\overline{S_{w e}}, \overline{f_{w e}}\right)$

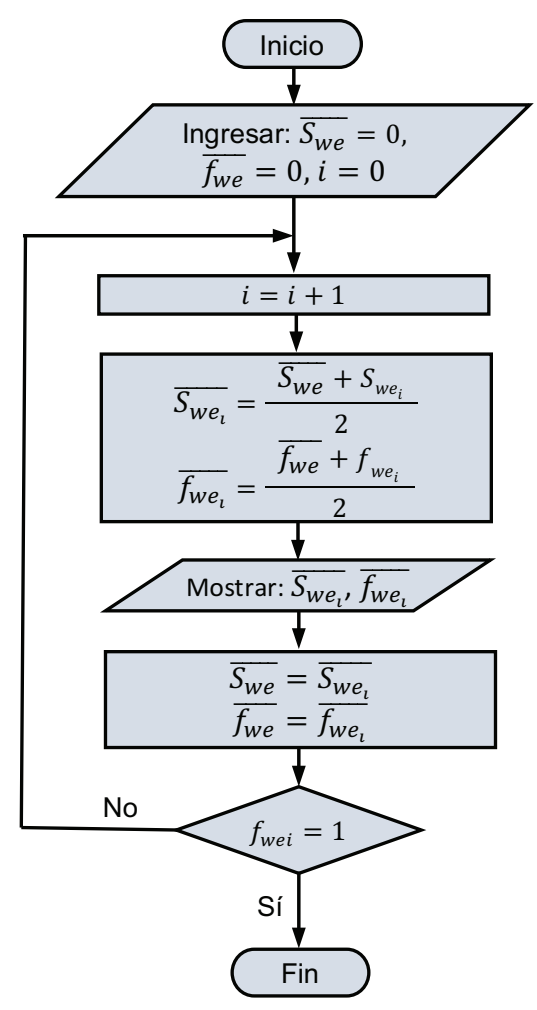

- Producción de petróleo $\left(N_{p d}\right)$

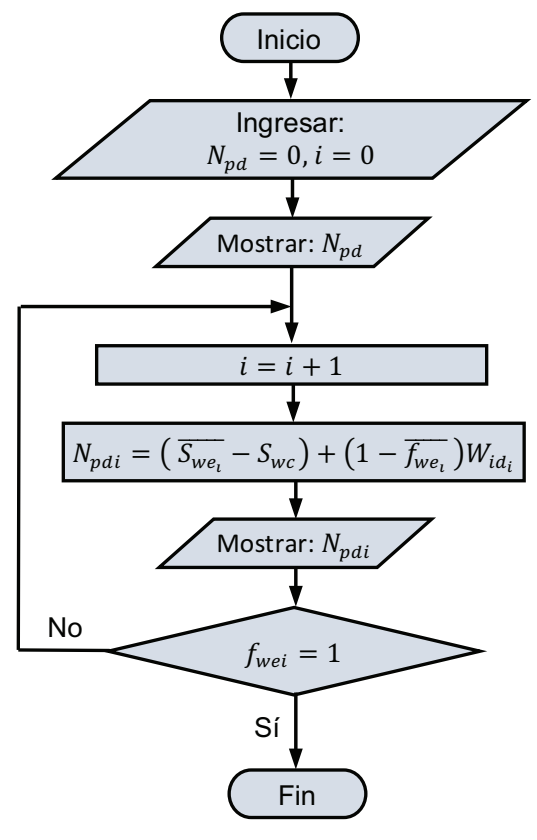


- Tiempo en días $(t)$

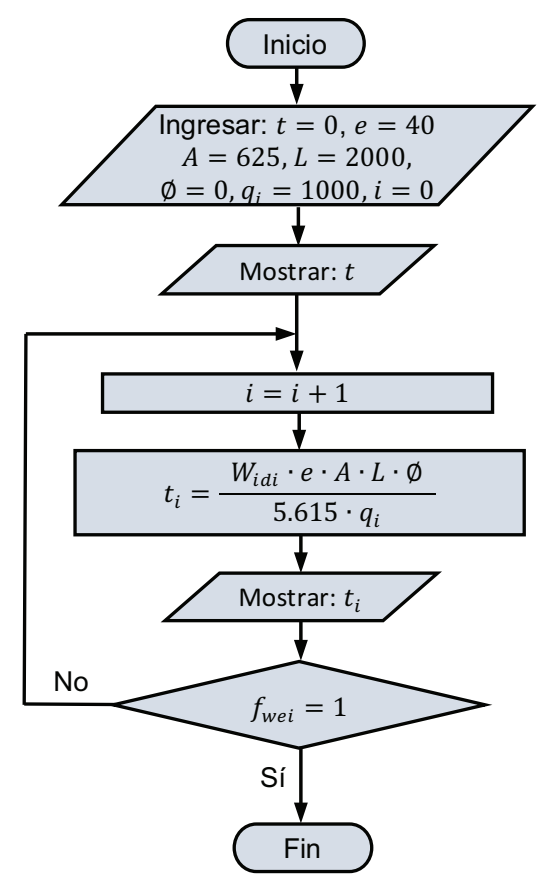

c) Flujo segregado

- Movilidad $(M)$ y agua acumulada inyectada ( $\left.\boldsymbol{W}_{\text {id(MOV) }}\right)$

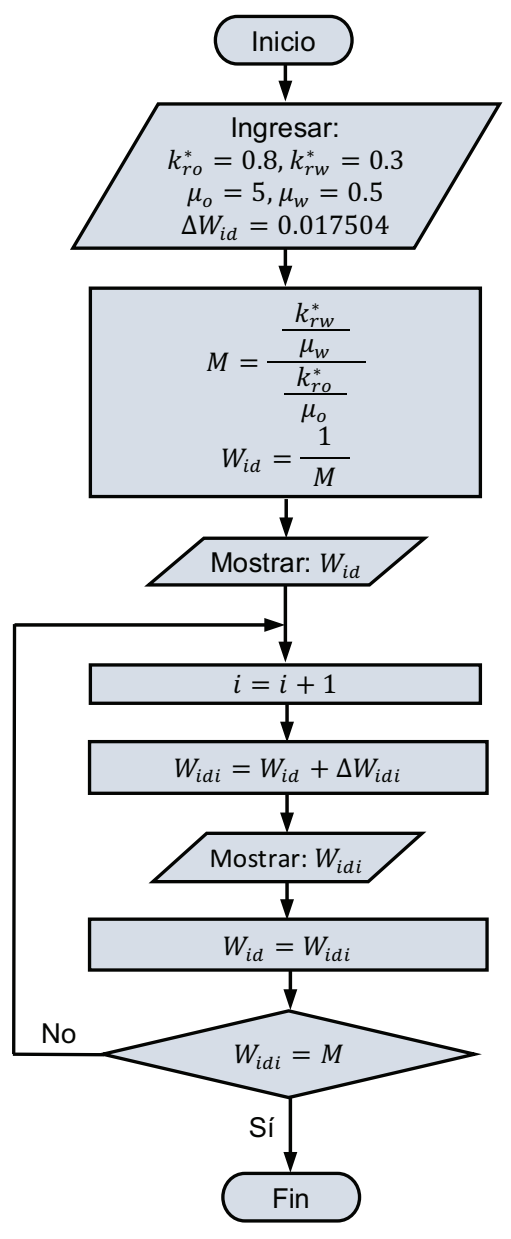

- Producción de petróleo $\left(N_{d p(M O V)}\right)$

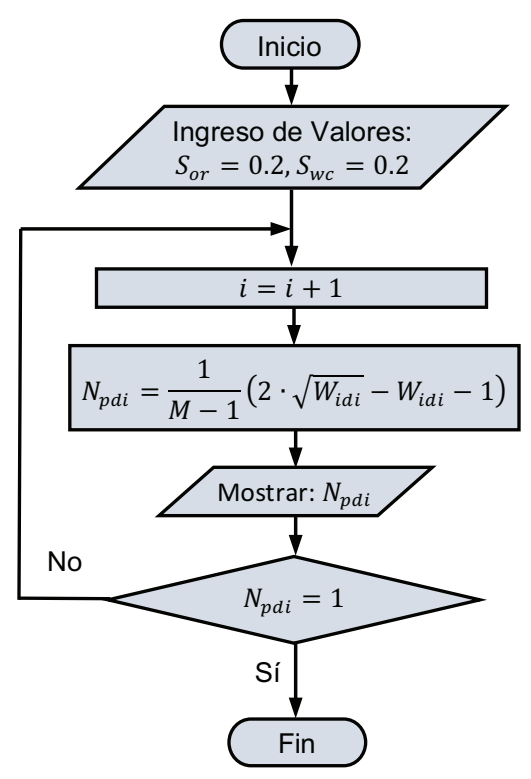

- Agua acumulada inyectada $\left(W_{i d}(P V)\right) y$ producción de petróleo $\left(N_{d p}(P V)\right)$

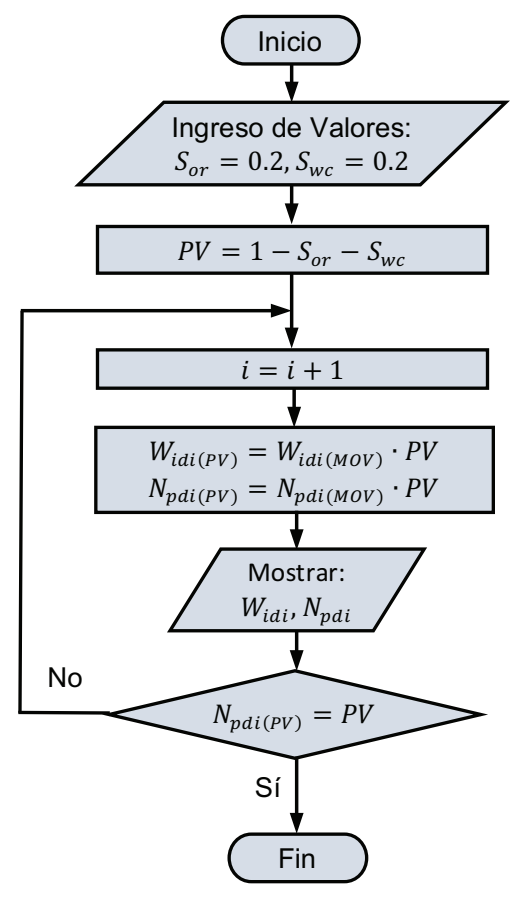


- Tiempo en días $(t)$

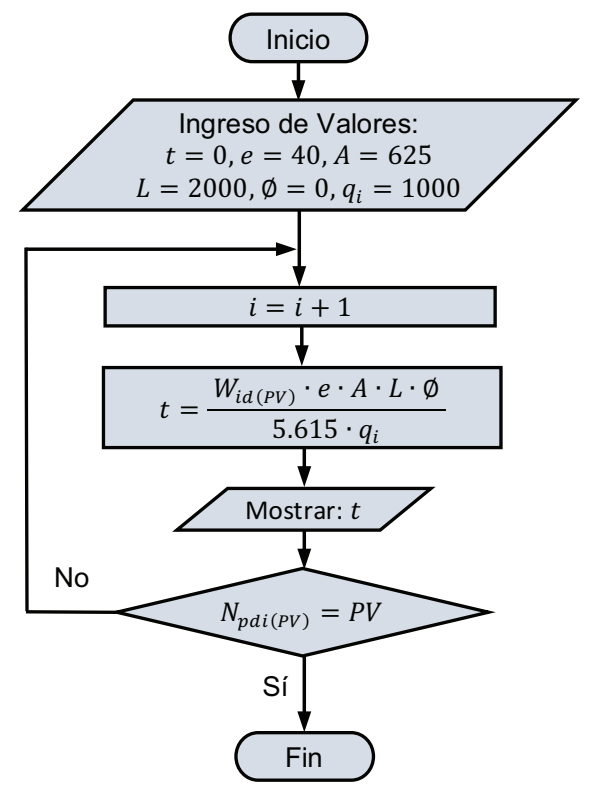

d) Perfiles segregado y difuso

- Tasa de inyección $\left(q_{i}\right)$ y agua acumulada $\left(W_{i}\right)$

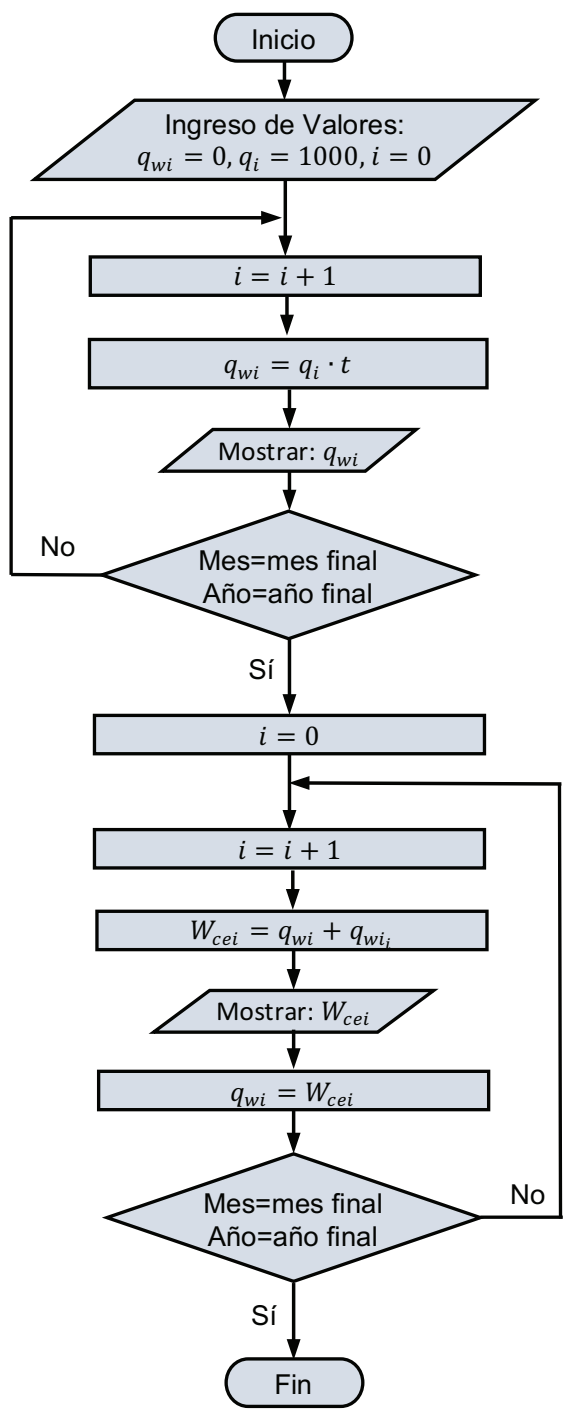

- Diagrama para días de acuerdo con los meses
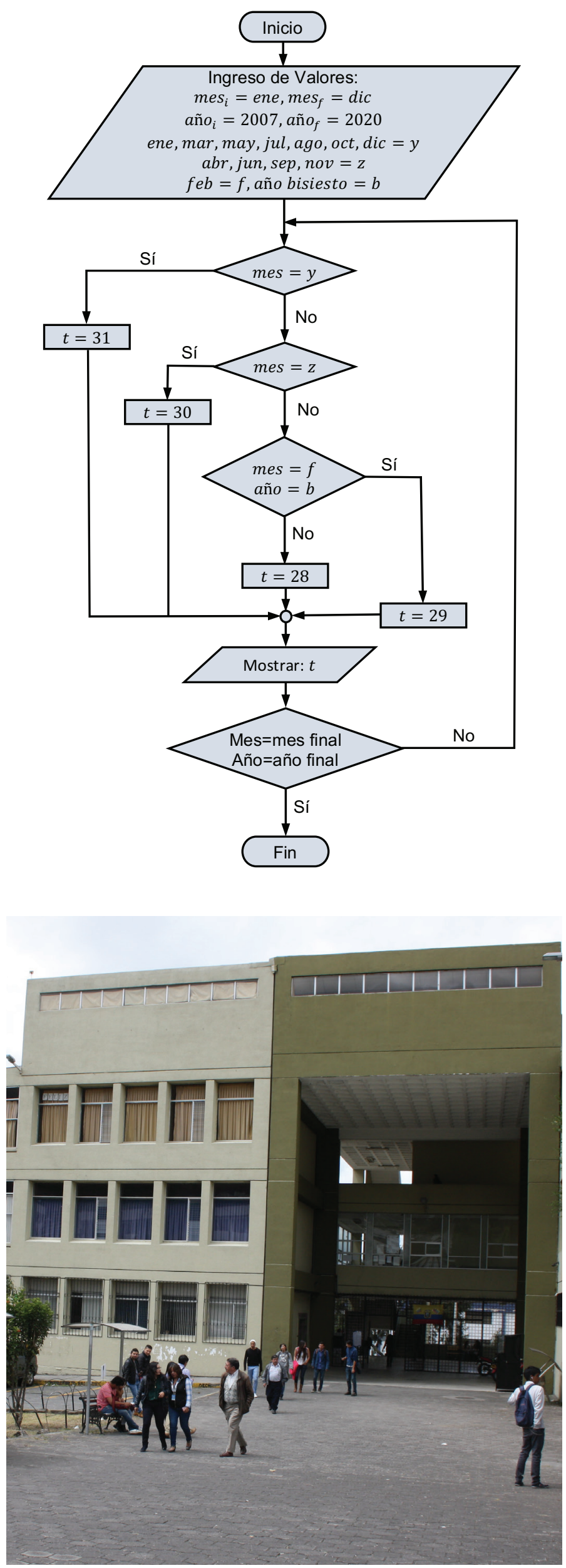
- Diagrama para caudal de petróleo y petróleo acumulado

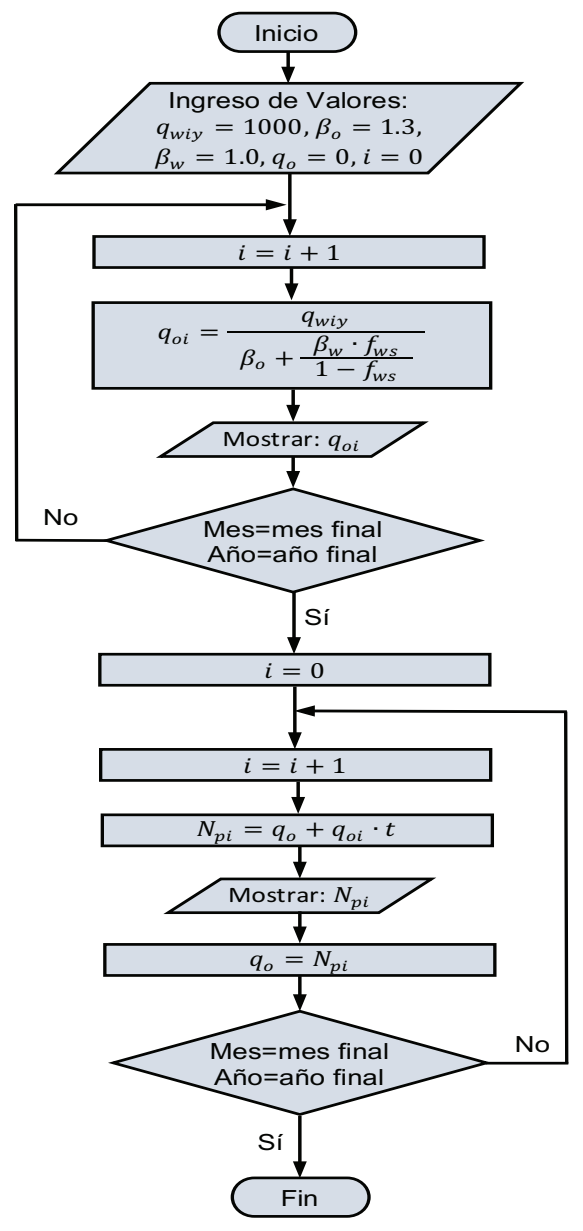

- Diagrama para petróleo original en sitio (OOIP) y factor de recobro

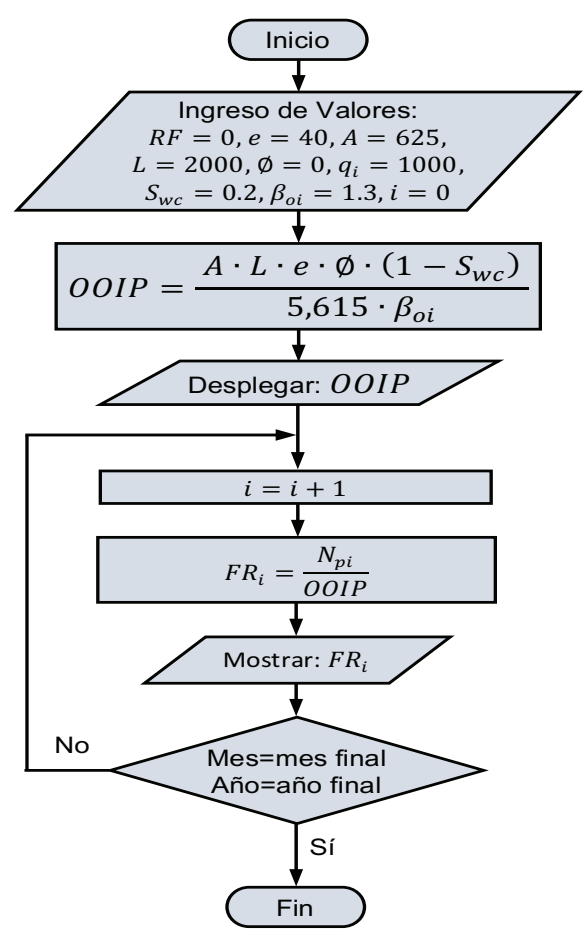

- Diagrama para caudal de agua y agua acumulada

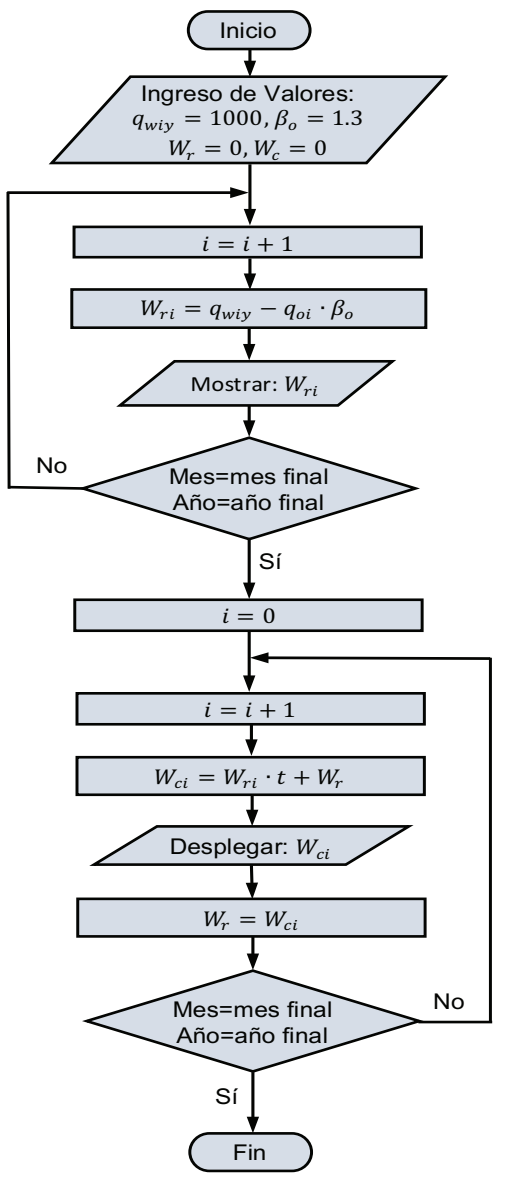

- Diagrama para relación agua petróleo (WOR) y corte de agua

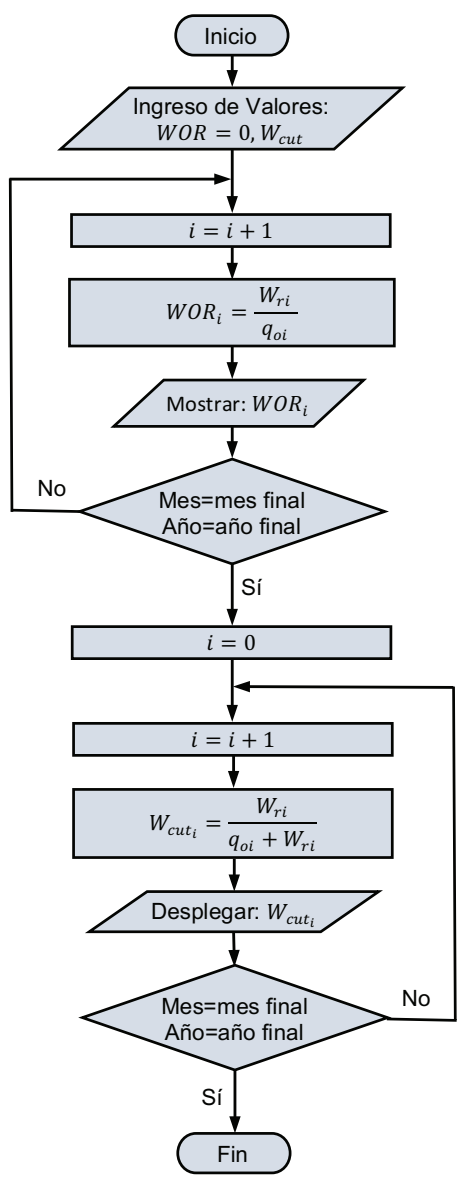




\section{Discusión}

Los resultados que se obtuvieron con el software WaterDrive y el método desarrollado se resumen en la tabla 3.

Tabla 3 Comparación de resultados obtenidos por software Water-Drive y por metodología

\begin{tabular}{|l|r|r|r|}
\hline \multicolumn{1}{|c|}{ Parámetro } & \multicolumn{1}{c|}{ WaterDrive } & \multicolumn{1}{c|}{ Método } & \% error \\
\hline OOIP (stb) & 986.4 & 986.4 & 0.00 \\
\hline Endpoint Mobility Ratio & 3.75 & 3.75 & 0.00 \\
\hline
\end{tabular}

\section{Flujo segregado}

\begin{tabular}{|l|r|r|r|}
\hline Critical WI rate [mstb/d] & 0.00 & 0.00 & 0.00 \\
Gravity Number & 0.00 & 0.00 & 0.00 \\
Displacement condition & Unstable & Inestable & \\
\hline Npd [bt] & 0.16 & 0.16 & 0.00 \\
Npd [ultimate] & 0.60 & 0.60 & 0.00 \\
Np, bbl [bt] & $197,279.1$ & $197,273.8$ & 0.00 \\
Np, bbl [ultimate] & $739,796.6$ & $739,776.7$ & 0.00 \\
\hline
\end{tabular}

Flujo difuso

\begin{tabular}{|l|r|r|r|}
\hline SF Mobility Ratio & 0.844259 & 0.8497 & 0.63 \\
Sw [bt] & 0.448485 & 0.45 & 0.34 \\
fw [bt] & 0.761821 & 0.7663 & 0.59 \\
Time, days [bt] & 522.84 & 516.1 & 1.28 \\
Npd [bt] & 0.326172 & 0.3252 & 0.29 \\
Npd [ultimate] & 0.600000 & 0.60 & 0.00 \\
Np, bbl [bt] & $402,168.3$ & $401,006.7$ & 0.29 \\
Np, bbl [ultimate] & $739,796.6$ & $739,776.7$ & 0.00 \\
\hline
\end{tabular}

De las comparaciones de resultados se destaca que para flujo segregado los valores son prácticamente idénticos.

Para flujo difuso, el error porcentual más alto fue de 1,28 por ciento en el cálculo del tiempo de ruptura. El resto de valores tienen un error porcentual menor al uno por ciento. Se puede considerar que estos errores serían el producto del cálculo de la tangente resultante de la ecuación de avance frontal con el flujo fraccional. Esta tangente fue calculada usando un programa externo (MatLab) que pudo haber incorporado errores.

Se estima que estos errores están en el rango teórico aceptable que pueden ser corregidas en versiones futuras de la metodología.

\section{Conclusiones}

La metodología creada en el libro Excel resultó compatible cuando se compararon estos resultados con los del software WaterDrive. Se obtuvo un error máximo de 1,28 por ciento en el flujo difuso durante el cálculo del tiempo de ruptura y un porcentaje de cero por ciento en los cálculos relacionados al flujo segregado.

El desarrollo de esta metodología prueba que es posible replicar un software compatible con programas realizados en países con mayor avance tecnológico. También permite continuar con el desarrollo del resto de módulos pertenecientes al software de recuperación secundaria.

\section{Agradecimientos}

Los autores agradecen a los colegas de la Facultad de Ingeniería en Geología, Minas, Petróleos, y Ambiental (Figempa) de la Universidad Central del Ecuador que realizaron contribuciones en la escritura del documento.

\section{Referencias bibliográficas}

Zitha, P., Felder, R., Zornes, D., Brown, K., \& Mohanty, K. (11 de July de 2011). www.spe.org/industry/increasing-hydrocarbon-recovery-factors.php. Recuperado el 19 de 10 de 2016, de www.spe.org/industry: www.spe.org/industry/ increasing-hydrocarbon-recovery-factors.php

Condor, J. A., Pinto, G. R., Achig, S., \& Romo, J. M. (2015). Identification of Enhanced Oil Recovery Potential in Ecuador. SPE Latin American and Caribbean Petroleum Engineering Conference. Quito: Society of Petroleum Engineers.

Petroleum Solutions. (29 de 09 de 2016). www.petroleumsolutions.co.uk/waterdrivemain.html. Recuperado el 29 de 09 de 2016, de www.petroleumsolutions.co.uk: http://www. petroleumsolutions.co.uk/waterdrivemain.html

Montalvo, P. S., \& Navarrete, J. F. (August de 2016). Desarrollo de un modelo predictivo para inyección de agua utilizando el método de Dake-Welge. Quito, Pichincha, Ecuador: Universidad Central del Ecuador

Dake, L. P. (2001). The Practice of Reservoir Engineering. Amsterdam: Springer.

Dake, L. P. (1998). Fundamentals of Reservoir Engineering. Amsterdam: Elsiever. 